\title{
Modelling and Analyzing the Potential Controls for Neospora caninum Infection in Dairy Cattle Using an Epidemic Approach
}

\author{
Yue Liu $\mathbb{D}^{1},{ }^{1}$ Ioannis Magouras, ${ }^{2}$ and Wing-Cheong Lo ${ }^{1}$ \\ ${ }^{1}$ Department of Mathematics, City University of Hong Kong, Tat Chee Avenue, Kowloon, Hong Kong, China \\ ${ }^{2}$ Department of Infectious Diseases and Public Health, City University of Hong Kong, Tat Chee Avenue, Kowloon, \\ Hong Kong, China \\ Correspondence should be addressed to Yue Liu; yue.liu@my.cityu.edu.hk
}

Received 22 February 2021; Revised 7 April 2021; Accepted 20 April 2021; Published 3 May 2021

Academic Editor: Toshikazu Kuniya

Copyright ( 2021 Yue Liu et al. This is an open access article distributed under the Creative Commons Attribution License, which permits unrestricted use, distribution, and reproduction in any medium, provided the original work is properly cited.

\begin{abstract}
Neospora caninum (N. caninum) infection, one of the major causes of abortions in dairy cattle, has brought a huge loss to farmers worldwide. In this study, we develop a six-compartment susceptible-infected model of $N$. caninum transmission which is later reduced to a two-equation system. Potential controls including medication, test-and-cull, and vaccination are proposed and analyzed, and the corresponding reproduction numbers are derived. The conditions for the global stabilities of disease-free and endemic equilibria are investigated with analytical solutions and geometric approach. Furthermore, uncertainty and sensitivity analysis shows that three control strategies are effective towards the varied environment, whereas the effectiveness of each measure highly depends on parameters related to control actions. Dynamics of reproduction numbers illustrate that disease elimination can be achieved by three types of controls: (1) adopting medication with medicine efficacy higher than 0.4 to prevent vertical transmission, (2) implementing test-and-cull with culling coverage larger than 0.3 , and (3) taking vaccine with coverage larger than 0.1. Numerical results suggest that preventive measures should at least include the prevention of access of other hosts, such as dogs, to cattle; otherwise, these control measures will lose effectiveness. Our presented study provides guidance for decisionmaking on $N$. caninum infected farm management.
\end{abstract}

\section{Introduction}

Neospora caninum (N. caninum) is a coccidian parasite which has been widely recognized as the predominant infection in dairy cattle [1-3]. The major transmission route is transplacental invasion of the embryo or fetus $[4,5]$. Moreover, cattle can also be infected horizontally by the seropositive ones within herd where $N$. caninum circulates endemically $[5,6]$ or other hosts from wildlife [7] by ingestion of food or drinking water contaminated by sporulated oocysts. Its transmission is associated with high abortion risk $[8,9]$, low milk yields due to adversely affected organ system functions, reduced weight gain, and premature culling [10]. The N. caninum infection in cattle is thus recognized as a major cause of abortion and economic loss to dairy industry worldwide $[1-3,11]$. Due to $N$. caninum, around 46.4 million cattle are reported to be at risk of abortion each year in different countries and more than US $\$ 1.3$ billion annual loss is caused in livestock [12]. As a result, all these losses stimulate a number of research works in several important areas, including improving our understanding of the transmission routes, quantifying the impact of $N$. caninum infection on production, and developing controls for preventing transmission.

Mathematical modelling has become a powerful tool in fully understanding and precisely describing the mechanisms of disease transmission [13-15]. In the context of $N$. caninum, French et al. [16] firstly proposed a system of nonlinear differential equations to describe the transmission of $N$. caninum and explored possible implementations by controlling key parameters to reduce infection. Larson et al. [17] used a 5-year simulation model to compare the control 
strategies and results show that, of the potential control strategies evaluated, testing the entire herd for $N$. caninum infection and excluding the daughters of seropositive dams provided the best economic return. Later on, Häsler et al. [18] used 12 age groups to depict the cattle population and investigated the impact of four controls, including test-based culling, medication, vaccination, and selective breeding of offspring, on population dynamics with computer simulations. Subsequently, Reichel et al. [19] developed a model using decision tree analysis to determine the relative cost/ benefit relationship of various control options of $N$. caninum infection. However, these works are mostly confined in numerical simulations to assess potential control options and much of the epidemiology of $N$. caninum transmission remains poorly understood. Therefore, we will establish an epidemiological model with key aspects of $N$. caninum transmission in dairy cattle. With the well-constructed model, we proceed to the assessment of potential control strategies from an epidemiological point of view.

The organization of our study is therefore as follows. In Section 2, we construct the mathematical model of $N$. caninum transmission incorporating vertical and horizontal infections with a system of nonlinear differential equations. The basic reproduction number is derived and global stability of both disease-free and endemic equilibria is proved. In Section 3, to prevent the N. caninum transmission, we consider three potential controls including medication, test-and-cull, and vaccination and give the corresponding control reproduction numbers. Global stabilities of the disease-free and endemic equilibrium points are investigated through an analytical solution or using a geometric approach. In Section 4, uncertainty and sensitivity analysis towards the varied environment is developed. We will discuss the effectiveness of three controls on the basis of reproduction number. Furthermore, we reveal the impact of the external source of infection. Finally, in Section 5, some conclusions are drawn.

\section{Methods}

2.1. Model Description and Construction. We present an epidemiological model based on the work $[16,18]$ to describe the dynamics of $N$. caninum-infected dairy cattle in Switzerland. The model is designed by dividing the farm into three age-groups according to their fertility status. It thus consists of offspring $(O)$, heifers $(H)$, and cows $(C)$ which represent the practical age $0-1,1-2$, and $\geq 2$, respectively. Each age group is further categorized into two epidemiological states: susceptible $(S)$ and infected $(I)$. Therefore, continuous-time variables $S_{O}(t), S_{H}(t)$, and $S_{C}(t)$ denote the number of three susceptible age groups and $I_{O}(t), I_{H}(t)$, and $I_{C}(t)$ denote the number of three infected age groups. In our model, the farm is taken as the fundamental epidemiological unit, and thus, the between-herd transmission is neglected. The farm is kept constant in size and open with replacement or culling, and only female animals are included.

Two major routes, vertical and horizontal infections, account for the N. caninum transmission in dairy herd. Vertical infection is the transfer of parasites from dams to offspring by transplacental route. Studies from different regions have provided strong evidence that this mode of transmission is highly efficient and the efficiency was reported to range from $81 \%$ to $95 \%[5,20,21]$. The calves born from infected mothers have a possibility to be susceptible without vertical infection rate $\rho_{v 1}$. Susceptible and infected animals have the same pregnancy rate $\alpha$ but the infected category has a two-to four-fold abortion risk of susceptible one due to $N$. caninum $[18,22]$. We assume a four-fold abortion risk in this study. On the contrary, the horizontal transmission process consists of within-herd level and outside-herd level (external source of infection). Potential sources of within-herd transmission include pooled colostrum or milk from infected cattle [23] and infected placenta or amniotic fluid or oocyst-contaminated feed which is therefore described as age-dependent infection force with a prevalence dependent factor $\zeta$. There is evidence suggesting that cattle may be infected by a definitive host, a dog or possibly other canids [24], and this external source of infection is denoted by a constant per-capita force $\sigma$.

Besides the vertical and horizontal transmissions, each age group has an outflow due to voluntary culling which includes natural death, sale, or slaughter. A proportion of animals will flow to the next age group due to aging. Furthermore, some animals will be bought from or sold to the market at a rate $\Lambda$ to balance the population. The population dynamics of $N$. caninum infected dairy cattle are described by the following equations:

$$
\left\{\begin{array}{l}
\frac{\mathrm{d} S_{O}}{\mathrm{~d} t}=\Lambda N+\alpha\left(1-\beta_{S}\right) S_{C}+\alpha\left(1-\beta_{I 1}\right)\left(1-\rho_{v 1}\right) I_{C}-\left(\delta_{S 1}+\rho_{h}\right) S_{O}-g S_{O} \\
\frac{\mathrm{d} S_{H}}{\mathrm{~d} t}=g S_{O}-\left(\delta_{S 2}+\rho_{h}\right) S_{H}-g S_{H} \\
\frac{\mathrm{d} S_{C}}{\mathrm{~d} t}=g S_{H}-\left(\delta_{S 3}+\rho_{h}\right) S_{C} \\
\frac{\mathrm{d} I_{O}}{\mathrm{~d} t}=\rho_{v 1} \alpha\left(1-\beta_{I 1}\right) I_{C}-\delta_{I 1} I_{O}+\rho_{h} S_{O}-g I_{O} \\
\frac{\mathrm{d} I_{H}}{\mathrm{~d} t}=g I_{O}-\delta_{I 2} I_{H}+\rho_{h} S_{H}-g I_{H} \\
\frac{\mathrm{d} I_{C}}{\mathrm{~d} t}=g I_{H}-\delta_{I 3} I_{C}+\rho_{h} S_{C}
\end{array}\right.
$$

where the horizontal transmission function $\rho_{h}=\zeta\left(I_{C} / S_{C}+\right.$ $\left.I_{C}\right)+\sigma$ and $S_{O}+S_{H}+S_{C}+I_{O}+I_{H}+I_{C}=N$ with $S_{O}>0$, $S_{H}>0, S_{C}>0, I_{O} \geq 0, I_{H} \geq 0$, and $I_{O} \geq 0$. To reduce the 
complexity without losing key features, we assume that offspring, heifers, and cows maintain a constant proportion $p, q$, and $r$ in the combined susceptible $(S)$ category and infected $(I)$ category, that is,

$$
\begin{aligned}
& S_{O}=p S, \\
& S_{H}=q S, \\
& S_{C}=r S, \\
& I_{O}=p I, \\
& I_{H}=q I, \\
& I_{C}=r I,
\end{aligned}
$$

where the values of $p, q$, and $r$ are later estimated by the proportions of offspring, heifers, and cows at the steady state of system (1). Then, the six-equation system (1) is reduced to a two-equation model with the horizontal transmission function $\rho_{h}=\zeta(I / N)+\sigma$. To keep a high-quality farm, mature animals inferior to genetic qualities would be removed by farmers each year. Removal could be the departure of animals that are surplus to herd requirement, producing low yield or avoiding accumulation. For immature animals (in age group 1 or 2 ), culling rates, $\delta_{S k}$ and $\delta_{I k}(k=1,2)$, are only due to mortality $\delta$. Whereas the culling rates of mature animals (in age group 3), $\delta_{S 3}$ and $\delta_{I 3}$, consist of basic mortality $\delta$ and varied removal rate $\epsilon$. It thus reflects that $\delta_{S 1}=\delta_{S 2}=\delta, \quad \delta_{S 3}=\delta+\varepsilon \quad$ and $\quad \delta_{I 1}=\delta_{I 2}=\delta, \quad \delta_{I 3}=\delta+\varepsilon$. Detailed flow diagram with six compartments, $S_{O}, S_{H}, S_{C}, I_{O}$, $I_{H}$, and $I_{C}$ describing the dynamics of $N$. caninum transmission among cattle is illustrated in Figure 1, and the interpretations of variables and parameters are shown in Table 1. It hence suffices to consider the reduced twoequation system:

$$
\left\{\begin{array}{l}
\frac{\mathrm{d} S}{\mathrm{~d} t}=\Lambda N+\alpha\left(1-\beta_{S}\right) r S+\alpha\left(1-\beta_{I 1}\right)\left(1-\rho_{v 1}\right) r I-(\delta+\varepsilon r) S-\rho_{h} S, \\
\frac{\mathrm{d} I}{\mathrm{~d} t}=\alpha \rho_{v 1}\left(1-\beta_{I 1}\right) r I-(\delta+\varepsilon r) I+\rho_{h} S,
\end{array}\right.
$$

where the reliability of this model reduction will be verified by numerical simulations later in Section 4. With the balanced population, the net growth of population should be 0 , and hence, the varied buying or selling rate is determined by $\Lambda=(\delta+\varepsilon r)-\left(\alpha\left(1-\beta_{S}\right) r S+\alpha\left(1-\beta_{I 1}\right) r I / N\right)$. Considering closed population $N=S(0)+I(0)$, it is not difficult to see that solution domain $\Omega=\left\{(S, I) \in \mathbb{R}_{+}^{2}: S(t)+I(t)=N\right\}$ is positively invariant and attracting all orbits for any nonnegative initial point. Thus, model (3) is mathematically and epidemiologically well posed in $\Omega$.

2.2. The Reproduction Number. In epidemiology, the basic reproduction number is one of the most important thresholds. It indicates the expected number of secondary cases caused by an infected individual during the infectious period with a fully susceptible population [27]. If it is larger than one, the disease will spread in the population; if it is smaller than one, the disease will die out. Therefore, the

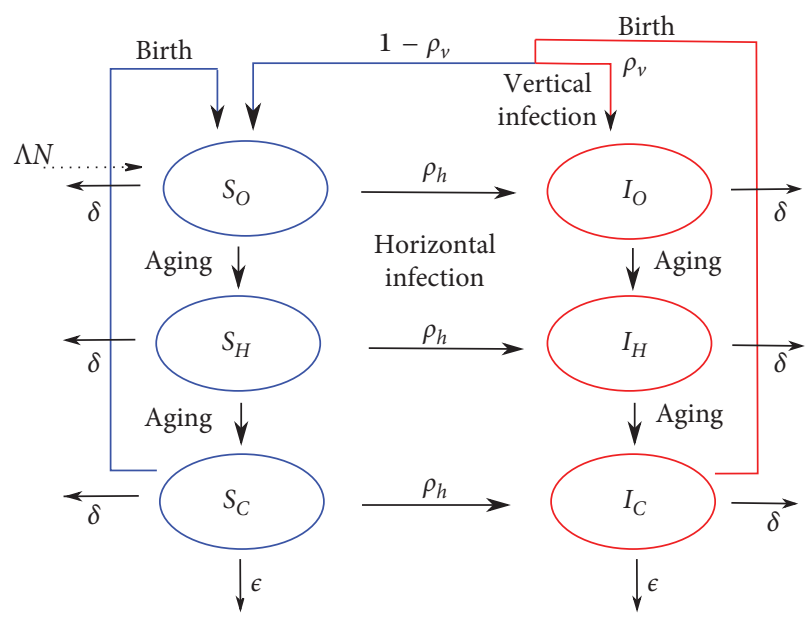

FIGURE 1: The flow diagram describing the dynamics of $N$. caninum infection in dairy cattle (dashed line: the varied buying or selling rate $\Lambda$ into the $S_{O}$ compartment). Subscripts $O, H$, and $C$ represent offspring, heifers, and cows, respectively.

reproduction number of controls can be used to assess the effectiveness of strategies. Due to the outside herd transmission term $\sigma$, which acts as an infection reservoir, there is no disease-free equilibrium. If a farm applies good external biosecurity, horizontal infection is only possible within herd. In the absence of an external infection source, i.e., $\sigma=0$, system (3) admits a disease-free equilibrium $E_{0}=(N, 0)$. We proceed with the Jacobian approach, and thus, it leads to the basic reproduction number:

$$
\mathscr{R}_{0}=\frac{\alpha \rho_{v 1}\left(1-\beta_{I 1}\right) r+\zeta}{\delta+\varepsilon r},
$$

where $(1 / \delta+\varepsilon r)$ interprets the lifespan of an infected cow and $\alpha \rho_{v 1}\left(1-\beta_{I 1}\right) r+\zeta$ interprets the number of secondary infections produced by a single infected cow per unit of time. Specifically, $\alpha \rho_{v 1}\left(1-\beta_{I 1}\right) r$ is the new infection from vertical transmission and $\zeta$ represents the new cases from the horizontal transmission.

Theorem 1. For system (3), $\mathscr{R}_{0}$ is defined as (4), and we have

(a) If $\mathscr{R}_{0}<1$, the disease-free equilibrium $E_{0}$ is globally asymptotically stable

(b) If $\mathscr{R}_{0}>1$, the disease-free equilibrium $E_{0}$ is unstable and there exists an endemic equilibrium $E_{*}=\left(S^{*}, I^{*}\right)$ with $S^{*}=\left((\delta+\varepsilon r)-\alpha \rho_{\nu 1}\left(1-\beta_{I 1}\right) r / \zeta\right) N \quad$ and $I^{*}=\left((\delta+\varepsilon r)\left(\mathscr{R}_{0}-1\right) / \zeta\right) N$ which is globally asymptotically stable

Proof. It is not difficult to see that system (3) can be analytically solved. The solution of infected group leads to $I(t)=$ $\left(\alpha \rho_{v 1}\left(1-\beta_{I 1}\right) r-(\delta+\varepsilon r)+\zeta / \quad C_{0} \exp \left[-\left(\alpha \rho_{v 1}\left(1-\beta_{I 1}\right) r-\right.\right.\right.$ $(\delta+\varepsilon r)+\zeta) t]+\zeta) N$, where $C_{0}$ is the constant determined by initial condition. With the derived control reproduction number $\mathscr{R}_{0}$ in equation (4), $I(t)$ can be written as $I(t)=$ $\left(\left(\mathscr{R}_{0}-1\right)(\delta+\varepsilon r) / \quad C_{0} \exp \left[-\left(\mathscr{R}_{0}-1\right)(\delta+\varepsilon r) t\right]+\zeta\right) N$, when $\mathscr{R}_{0}<1$ and $I(t) \longrightarrow 0$ as $t \longrightarrow \infty$; when $\mathscr{R}_{0}>1$, 
TABLE 1: Description of parameters and variables used in the model.

\begin{tabular}{|c|c|c|c|c|}
\hline Notation & Definition & Unit & Value & Reference \\
\hline$\Lambda$ & Buying or selling rate of animals & Year $^{-1}$ & - & - \\
\hline$\alpha$ & Pregnancy rate & Year $^{-1}$ & 0.30 & {$[18,25]$} \\
\hline$\beta_{S}$ & Abortion rate of susceptible cow & Unitless & 0.02 & {$[18]$} \\
\hline$\beta_{I 1}$ & Abortion rate of infected cow & Unitless & 0.08 & {$[18,22]$} \\
\hline$\rho_{h}$ & Horizontal infection rate & Year $^{-1}$ & - & - \\
\hline$\rho_{v 1}$ & Vertical infection rate of infected cow & Unitless & 0.90 & {$[18,20]$} \\
\hline$\zeta$ & Prevalence dependent factor & Unitless & 0.028 & {$[16,18]$} \\
\hline$p$ & Proportion of offspring & Unitless & 0.25 & Estimated \\
\hline$q$ & Proportion of heifers & Unitless & 0.25 & Estimated \\
\hline$r$ & Proportion of cows & Unitless & 0.50 & Estimated \\
\hline$\delta$ & Mortality rate & Year $^{-1}$ & 0.10 & [18] \\
\hline$\epsilon$ & Removal rate & Year $^{-1}$ & 0.095 & Assumed \\
\hline$\eta$ & Recovery rate of dams & Year $^{-1}$ & 0.60 & Assumed \\
\hline$\sigma$ & External transmission coefficient & Year $^{-1}$ & $0-0.07$ & Assumed \\
\hline$c$ & Culling proportion in test-and-cull & Unitless & 0.70 & Assumed \\
\hline$\gamma_{1}$ & Specificity of serological test & Unitless & 0.98 & [18] \\
\hline$\gamma_{2}$ & Sensitivity of serological test & Unitless & 0.96 & {$[18]$} \\
\hline$\phi$ & Coverage rate of vaccination & Unitless & 0.50 & Assumed \\
\hline$\theta$ & Efficacy of vaccination & Unitless & 0.90 & {$[18,26]$} \\
\hline$\beta_{I 2}$ & Abortion rate of vaccinated infected cow & Unitless & 0.06 & {$[18,26]$} \\
\hline$\rho_{v 2}$ & Vertical infection rate of vaccinated infected cow & Unitless & 0.40 & Assumed \\
\hline
\end{tabular}

$I(t) \longrightarrow\left(\left(\mathscr{R}_{0}-1\right)(\delta+\varepsilon r) / \zeta\right) N$ as $t \longrightarrow \infty$. Therefore, it concludes that $E_{0}$ is globally asymptotically stable whenever $\mathscr{R}_{0}<1$, and $E_{*}$ is globally asymptotically stable whenever $\mathscr{R}_{0}>1$. The proof is completed.

\section{Stability Analysis of Each Control Strategy}

To control the spread of $N$. caninum, different control measures that intrinsically lie in reducing the vertical transmission or the risk of horizontal infection of $N$. caninum have been suggested. To date, three kinds of interventions including medication, test-and-cull, and vaccination are typically recommended [18, 19, 28, 29]. We construct a control model first and then compute the control reproduction number. Global stabilities of disease-free and endemic equilibria are further investigated whether using an analytical solution or a geometric approach [30, 31].
3.1. Medication. One of the promising strategies for controlling bovine neosporosis is through chemotherapy on newborn offspring. Currently, no available medicine has been proved to be effective. However, the treatment can also be discussed in advance since it may be available at a later stage. An experimental chemotherapeutic treatment study [32] found that following treatment of calves with ponazuril, parasite loads were reduced in the brain. An experiment with infected mice [33] supported that treatment could considerably reduce the diaplacental passage of the parasite from dam to the fetal brain. Therefore, we assume that chemotherapy was able to block transplacental transmission of $N$. caninum in cattle. All calves born from infected mothers will be treated without previous testing, and thus, chemotherapy treatment on neonatal calves from infected dams had a proportion $\eta$ to recover. This portion of recovered calves would flow into noninfected category. As for the population structure, only the number of new birth flowing into the susceptible group will change:

$$
\left\{\begin{array}{l}
\frac{\mathrm{d} S}{\mathrm{~d} t}=\Lambda N+\alpha\left(1-\beta_{S}\right) r S+\alpha\left(1-\beta_{I 1}\right)\left(1-\rho_{v 1}(1-\eta)\right) r I-(\delta+\varepsilon r) S-\rho_{h} S \\
\frac{\mathrm{d} I}{\mathrm{~d} t}=\alpha \rho_{v 1}(1-\eta)\left(1-\beta_{I 1}\right) r I-(\delta+\varepsilon r) I+\rho_{h} S .
\end{array}\right.
$$

If there is no medicine treatment, i.e., $\eta=0$, system (5) is reduced to the baseline case (3). The control reproduction number $\mathscr{R}_{0}^{m}$ of medication is

$$
\mathscr{R}_{0}^{m}=\frac{\alpha \rho_{v 1}(1-\eta)\left(1-\beta_{I 1}\right) r+\zeta}{\delta+\varepsilon r} .
$$

Compared to $\mathscr{R}_{0}$, a reduction term $(1-\eta)$ is multiplied to vertical transmission and thus $\mathscr{R}_{0}^{m} \leq \mathscr{R}_{0}$. When $\mathscr{R}_{0}^{m}>1$, the system admits an endemic equilibrium $E_{*}^{m}=\left(S^{*}, I^{*}\right)$ with $S^{*}=\left((\delta+\varepsilon r)-\alpha \rho_{v 1}(1-\eta)\left(1-\beta_{I 1}\right) / \zeta\right) N \quad$ and $I^{*}=\left(\left(\mathscr{R}_{0}^{m}-1\right)(\delta+\varepsilon r) / \zeta\right) N$. Based on Theorem 1 and $\rho_{v 1}$ 
being replaced by $\rho_{v 1}(1-\eta)$, the following stability result can be obtained.

Corollary 1. For system (5), $\mathscr{R}_{0}^{m}$ is defined as (6), and we have

(a) If $\mathscr{R}_{0}^{m}<1$, the disease-free equilibrium $E_{0}^{m}$ is globally asymptotically stable

(b) If $\mathscr{R}_{0}^{m}>1$, the disease-free equilibrium $E_{0}^{m}$ is unstable and there exists an endemic equilibrium $E_{*}^{m}=\left(S^{*}, I^{*}\right)$ with $S^{*}=\left((\delta+\varepsilon r)-\alpha \rho_{v 1}(1-\eta)(1-\right.$ $\left.\left.\beta_{I 1}\right) / \zeta\right) N, \quad I^{*}=\left(\left(\mathscr{R}_{0}^{m}-1\right)(\delta+\varepsilon r) / \zeta\right) N$ which is globally asymptotically stable
3.2. Test-and-Cull. Test-based culling is an efficacious and practical intervention [20]. Currently, the diagnostic test is conducted by blood sampling which is combined with the regular bulk-milk monitoring system [34]. Once animals are tested seropositive, they will be either slaughtered or sold. With this control strategy, the culling is regarded as a continuous action and the culling rate is set to $c$. The susceptible individuals that were wrongly identified $\left(1-\gamma_{1}\right)$ seropositive by test and the infected individuals that were correctly classified $\left(\gamma_{2}\right)$ are identified as seropositive. The dynamics of the model considering test-based culling are described as follows:

$$
\left\{\begin{array}{l}
\frac{\mathrm{d} S}{\mathrm{~d} t}=\Lambda N+\alpha\left(1-\beta_{S}\right) r S+\alpha\left(1-\beta_{I 1}\right)\left(1-\rho_{v 1}\right) r I-\left[\delta+c\left(1-\gamma_{1}\right) r\right] S-\rho_{h} S \\
\frac{\mathrm{d} I}{\mathrm{~d} t}=\alpha \rho_{v 1}\left(1-\beta_{I 1}\right) r I-\left(\delta+c \gamma_{2} r\right) I+\rho_{h} S
\end{array}\right.
$$

where the varied buying or selling rate $\Lambda=\left(\left[\delta+c\left(1-\gamma_{1}\right) r\right] S+\left(\delta+c \gamma_{2} r\right) I-\left[\alpha\left(1-\beta_{S}\right) r S+\alpha(1-\right.\right.$ $\left.\left.\left.\beta_{I 1}\right) r I\right] / N\right)$, to keep a closed population.

If there is no any testing-based culling and replacement, i.e., $\Lambda=0, \gamma_{1}=0$, and $\gamma_{2}=1$, it thus corresponds to baseline model (3). In general, the control reproduction number of test-and-cull case $\mathscr{R}_{0}^{t}$ is

$$
\mathscr{R}_{0}^{t}=\frac{\alpha \rho_{v 1}\left(1-\beta_{I 1}\right) r+\zeta}{\delta+c \gamma_{2} r},
$$

where $\left(1 / \delta+c \gamma_{2} r\right)$ interprets the lifespan of an infected cow and $\alpha \rho_{v 1}\left(1-\beta_{I 1}\right) r$ is the new infection coming from vertical transmission and $\zeta$ represents the new cases from horizontal transmission. When $1<\mathscr{R}_{0}^{t}<1+\left(\zeta / \delta+c \gamma_{2} r\right)$, there exists an endemic equilibrium $E_{*}^{t}=\left(S^{*}, I^{*}\right)$ with $S^{*}=(N / \zeta)(1+$ $\left.\left(\zeta / \delta+c \gamma_{2} r\right)-\mathscr{R}_{0}^{t}\right)\left(\delta+c \gamma_{2} r\right)$ and $I^{*}=(N / \zeta)\left(\mathscr{R}_{0}^{t}-1\right)(\delta+$ $\left.c \gamma_{2} r\right)$.

Theorem 2. For system (7), $\mathscr{R}_{0}^{t}$ is defined as (8), and we have

(a) If $\mathscr{R}_{0}^{t}<1$, the disease-free equilibrium $E_{0}^{t}$ is globally asymptotically stable

(b) If $1<\mathscr{R}_{0}^{t}<1+\left(\zeta / \delta+c \gamma_{2} r\right)$, the disease-free equilibrium $E_{0}^{t}$ is unstable and there exists an endemic equilibrium $E_{*}^{t}=\left(S^{*}, I^{*}\right)$ with $S^{*}=(N / \zeta)(1+(\zeta / \delta$ $\left.\left.+c \gamma_{2} r\right)-\mathscr{R}_{0}^{t}\right)\left(\delta+c \gamma_{2} r\right), \quad I^{*}=(N / \zeta)\left(\mathscr{R}_{0}^{t}-1\right)(\delta+$ $\left.c \gamma_{2} r\right)$ which is globally asymptotically stable
Proof. The solution of the infected group in system (7) gives $I_{\text {test-and-cull }}(t)=\left(\left(\mathscr{R}_{0}^{t}-1\right)\left(\delta+c \gamma_{2} r\right) / C_{0} \exp \left[-\left(\mathscr{R}_{0}^{t}-1\right)(\delta+\right.\right.$ $\left.\left.\left.c \gamma_{2} r\right) t\right]+\zeta\right) N$, where $C_{0}$ is determined by initial condition. When $\mathscr{R}_{0}^{t}<1, \quad I_{\text {test-and-cull }}(t) \longrightarrow 0$ as $t \longrightarrow \infty$; when $\mathscr{R}_{0}^{t}>1, \quad I_{\text {test-and-cull }}(t) \longrightarrow(N / \zeta)\left(\mathscr{R}_{0}^{t}<1\right)\left(\delta+c \gamma_{2} r\right) \quad$ as $t \longrightarrow \infty$. Therefore, it concludes that $E_{0}^{t}$ is globally asymptotically stable whenever $\mathscr{R}_{0}^{t}<1$, and $E_{*}^{t}$ is globally asymptotically stable whenever $1<\mathscr{R}_{0}^{t}<1+\left(\zeta / \delta+c \gamma_{2} r\right)$. The proof is completed.

3.3. Vaccination. To study control through vaccinating, two compartments, susceptible vaccinees $(V)$ and infected vaccinees $(W)$, will be added to the baseline case. Susceptible and infected individuals were vaccinated at a yearly coverage rate $\phi$ and vaccine efficacy was assumed to be $\theta$. A life-long time immunity is assumed. If the individual was susceptible, it became susceptible vaccinee $(V)$ at the rate $\phi \theta$ after the successful vaccination and it could not become infected due to the protection by the vaccine. If the individual was infected, it became infected vaccine $(W)$ at the rate $\phi \theta$ after the successful vaccination. Susceptible vaccines $(V)$ have the same pregnancy rate $\alpha$, abortion rate $\beta_{S}$, death rate $\delta$, and removal rate $\epsilon$ as susceptible individuals but are unable to be infected. It was assumed that the vaccine could reduce the risk of abortion and the vertical transmission from dam to offspring. Thus, the infected vaccinees $(W)$ have a lower abortion rate $\beta_{I 2}\left(\beta_{I 2}<\beta_{I 1}\right)$ compared to infected animals since vaccinated infected cows will be protected from 
$N$. caninum-caused abortion. Moreover, the vertical transmission rate $\rho_{v 2}\left(\rho_{v 2}<\rho_{v 1}\right)$ is also reduced while other characteristics are the same as $I$. The dynamic model with vaccination is

$$
\left\{\begin{array}{l}
\frac{\mathrm{d} S}{\mathrm{~d} t}=\Lambda N+\alpha\left(1-\beta_{S}\right) r S+\alpha\left(1-\beta_{I 1}\right)\left(1-\rho_{\nu 1}\right) r I+\alpha\left(1-\beta_{S}\right) r V+\alpha\left(1-\beta_{I 2}\right)\left(1-\rho_{v 2}\right) r W-(\delta+\varepsilon r) S-\rho_{h} S-\phi \theta S, \\
\frac{\mathrm{d} I}{\mathrm{~d} t}=\alpha \rho_{v 1}\left(1-\beta_{I 1}\right) r I+\alpha \rho_{v 2}\left(1-\beta_{I 2}\right) r W-(\delta+\varepsilon r) I+\rho_{h} S-\phi \theta I, \\
\frac{\mathrm{d} V}{\mathrm{~d} t}=\phi \theta S-(\delta+\varepsilon r) V, \\
\frac{\mathrm{d} W}{\mathrm{~d} t}=\phi \theta I-(\delta+\varepsilon r) W .
\end{array}\right.
$$

If $\phi=0, \theta=0$ which means that no vaccine has been taken, $\lim _{t \longrightarrow \infty} V(t)=0$, and $\lim _{t \rightarrow \infty} W(t)=0$, model (9) is reduced to baseline case (3). Similarly, the buying or selling rate of animals is allowed to vary to maintain population with size $N=S(t)+I(t)+V(t)+W(t)$, and therefore, it yields $\quad \Lambda=(\delta+\varepsilon r)-\left(\alpha\left(1-\beta_{S}\right) r(S+V)+\alpha\left(1-\beta_{I 1}\right) r I+\right.$ $\left.\alpha\left(1-\beta_{I 2}\right) r W / N\right)$ in the positively invariant region $\Omega=\left\{(S, I, V, W) \in \mathbb{R}_{+}^{4}: S(t)+I(t)+V(t)+W(t)=N\right\}$. It can be seen that model (9) owns a disease-free point $E_{0}^{v}=$ $\left(S^{0}, 0, V^{0}, 0\right) \quad$ with $S^{0}=((\delta+\varepsilon r) N / \delta+\varepsilon r+\phi \theta) \quad$ and $V^{0}=(\phi \theta N / \delta+\varepsilon r+\phi \theta)$. Following the next generation matrix approach [27], we obtain the $\mathscr{F}$ interpreting the production rate of new infections and the $\mathscr{V}$ representing the remaining transitional terms:

$$
\begin{aligned}
& \mathscr{F}=\left[\begin{array}{c}
\alpha \rho_{v 1}\left(1-\beta_{I 1}\right) r I+\alpha \rho_{v 2}\left(1-\beta_{I 2}\right) r W+\rho_{h} S \\
\phi \theta I
\end{array}\right], \\
& \mathscr{V}=\left[\begin{array}{c}
(\delta+\varepsilon r) I+\phi \theta I \\
(\delta+\varepsilon r) W
\end{array}\right] .
\end{aligned}
$$

Then, the derivatives of $\mathscr{F}$ and $\mathscr{V}$ evaluated at diseasefree equilibrium $E_{0}^{v}$, respectively, give $F\left(E_{0}^{v}\right)$ and $V\left(E_{0}^{v}\right)$ :

$$
\begin{aligned}
& F\left(E_{0}^{v}\right)=\left[\begin{array}{cc}
\alpha \rho_{v 1}\left(1-\beta_{I 1}\right) r+\zeta \frac{S^{0}}{N} & \alpha \rho_{v 2}\left(1-\beta_{I 2}\right) r \\
\phi \theta & 0
\end{array}\right], \\
& V\left(E_{0}^{v}\right)=\left[\begin{array}{cc}
\delta+\varepsilon r+\phi \theta & 0 \\
0 & \delta+\varepsilon r
\end{array}\right] .
\end{aligned}
$$

Thus, the control reproduction number $\mathscr{R}_{0}^{v}$ given by the spectral radius of $F\left(E_{0}^{v}\right) V\left(E_{0}^{v}\right)^{-1}$ is

$$
\mathscr{R}_{0}^{v}=\frac{\alpha \rho_{v 1}\left(1-\beta_{I 1}\right) r}{\delta+\varepsilon r+\phi \theta}+\frac{\zeta(\delta+\varepsilon r)}{(\delta+\varepsilon r+\phi \theta)^{2}}+\frac{\phi \theta}{\delta+\varepsilon r+\phi \theta} \frac{\alpha \rho_{v 2}\left(1-\beta_{I 2}\right) r}{\delta+\varepsilon r},
$$

where $\mathscr{R}_{0}^{v}$ consists of the number of secondary infections produced by an infected cow and an infected vaccine. The first two terms give the new infection cases in infected group $I$ from vertical and horizontal transmission routes, respectively. The third term, which interprets the secondary cases produced by an infected vaccinee $W$, has two components. $(\phi \theta / \delta+\varepsilon r+\phi \theta)$ is the number of infected cow flowing to infected vaccine group $(W)$ in the unit time, and infected vaccine generates $\alpha \rho_{\nu 2}\left(1-\beta_{I 2}\right) r$ infections during the life time $(1 / \delta+\varepsilon r)$. Moreover, system (9) has an endemic equilibrium $E_{*}^{v}=\left(S^{*}, I^{*}, V^{*}, W^{*}\right)$ with $S^{*}=(N / \zeta)(\delta+\varepsilon r+$ $\phi \theta)\left[1+\left(\zeta(\delta+\varepsilon r) /(\delta+\varepsilon r+\phi \theta)^{2}\right)-\mathscr{R}_{0}^{v}\right], I^{*}=(\delta+\varepsilon r / \delta+$ $\varepsilon r+\phi \theta) N-S^{*}$ which is equivalent to $I^{*}=(N / \zeta)(\delta+\varepsilon r+\phi$ $\theta)\left(\mathscr{R}_{0}^{v}-1\right), V^{*}=(\phi \theta / \delta+\varepsilon r) S^{*}$, and $W^{*}=(\phi \theta / \delta+\varepsilon r) I^{*}$. Thus, the endemic equilibrium $\left(S^{*}, I^{*}, V^{*}, W^{*}\right)$ exists whenever $1<\mathscr{R}_{0}^{v}<1+\left(\zeta(\delta+\varepsilon r) /(\delta+\varepsilon r+\phi \theta)^{2}\right)$.

Lemma 1. If $\mathscr{R}_{0}^{v}<1$, system (9) admits the disease-free equilibrium $E_{0}^{v}=\left(S^{0}, 0, V^{0}, 0\right)$. If $1<\mathscr{R}_{0}^{v}<1+(\zeta(\delta+\varepsilon r) /$ $\left.(\delta+\varepsilon r+\phi \theta)^{2}\right)$, the system has the unique endemic equilibrium $E_{*}^{v}=\left(S^{*}, I^{*}, V^{*}, W^{*}\right)$ with $S^{*}=(N / \zeta)(\delta+\varepsilon r+\phi \theta)$ $\left[1+\left(\zeta(\delta+\varepsilon r) /(\delta+\varepsilon r+\phi \theta)^{2}\right)-\mathscr{R}_{0}^{v}\right], \quad I^{*}=(N / \zeta)(\delta+\varepsilon r+$ $\phi \theta)\left(\mathscr{R}_{0}^{v}-1\right), V^{*}=(\phi \theta / \delta+\varepsilon r) S^{*}$, and $W^{*}=(\phi \theta / \delta+\varepsilon r) I^{*}$.

Next, we show the global stability of disease-free point $E_{0}^{v}$ following the comparison theorem developed by [35], and further, we investigate the global stability of endemic equilibrium $E_{*}^{v}$ with the geometric approach [30, 31], which was adopted by many researchers (see $[36,37])$. Using the relation $S(t)=N-I(t)-V(t)-W(t)$, one shall reduce (9) to the equivalent system: 


$$
\left\{\begin{array}{l}
\frac{\mathrm{d} I}{\mathrm{~d} t}=\alpha \rho_{v 1}\left(1-\beta_{I 1}\right) r I+\alpha \rho_{v 2}\left(1-\beta_{I 2}\right) r W-(\delta+\varepsilon r) I+\zeta \frac{I}{N}(N-I-V-W)-\phi \theta I, \\
\frac{\mathrm{d} V}{\mathrm{~d} t}=\phi \theta(N-I-V-W)-(\delta+\varepsilon r) V, \\
\frac{\mathrm{d} W}{\mathrm{~d} t}=\phi \theta I-(\delta+\varepsilon r) W
\end{array}\right.
$$

where the solution domain $\Gamma=\left\{(I, V, W) \in \mathbb{R}_{+}^{3}: I(t)+V(t)+W(t) \leq N\right\}$ is positively invariant with respect to system (13). Following CastilloChavez [35], system (13) is rewritten in the form

$$
\left\{\begin{array}{l}
\frac{\mathrm{d} X}{\mathrm{~d} t}=F(X, \mathbf{Y}), \\
\frac{\mathrm{d} \mathbf{Y}}{\mathrm{d} t}=G(X, \mathbf{Y}), \quad \text { with } G(X, \mathbf{0})=0,
\end{array}\right.
$$

where $X$ denotes the number of uninfected individuals and $Y$ denotes the number of infected individuals including $I$ and $W$. Thus, $X=V$ and $\mathbf{Y}=[I, W]^{T}$. Two conditions (H1) and (H2) below must be satisfied to guarantee the global asymptotic stability of $\left(X^{0}, \mathbf{0}\right)$ :

(H1) For $(\mathrm{d} X / \mathrm{d} t)=F(X, \mathbf{0}), X^{0}$ is globally stable

(H2) $G(X, \mathbf{Y})=-A \mathbf{Y}-\widehat{G}(X, \mathbf{Y}), \quad \widehat{G}(X, \mathbf{Y}) \geq 0 \quad$ for $(X, \mathbf{Y}) \in \Gamma$, where $A$ is an $M$ matrix

Lemma 2 (see [36]). The fixed point $\left(X^{0}, \mathbf{0}\right)$ is globally asymptotically stable provided that assumptions (H1) and (H2) are satisfied.

Theorem 3. The disease-free equilibrium $E_{0}^{v}$ is globally asymptotically stable if $\mathscr{R}_{0}^{v}<1-\left(\zeta \phi \theta /(\delta+\varepsilon r+\phi \theta)^{2}\right)$.

Proof. Let $X=V \quad$ and $\quad \mathbf{Y}=[I, W]^{T}$. Then, $(d X / d t)=\phi \theta(N-I-V-W)-(\delta+\varepsilon r) V \quad$ and $F(X, \mathbf{0})=\phi \theta(N-V)-(\delta+\varepsilon r) V$. As $t \longrightarrow \infty, V \longrightarrow V^{0}$. Hence, $X^{0}=V^{0}$ is globally asymptotically stable. Furthermore, in $G(X, \mathbf{Y})=-A \mathbf{Y}-\widehat{G}(X, \mathbf{Y})$, we have

$$
\begin{aligned}
A & =\left[\begin{array}{cc}
-\left(\alpha \rho_{v 1}\left(1-\beta_{I 1}\right) r+\zeta\right)+(\delta+\varepsilon r+\phi \theta) & -\alpha \rho_{v 2}\left(1-\beta_{I 2}\right) r \\
-\phi \theta & (\delta+\varepsilon r)
\end{array}\right], \\
\widehat{G}(X, \mathbf{Y}) & =\left[\begin{array}{c}
\zeta \frac{I}{N}(I+V+W) \\
0
\end{array}\right] .
\end{aligned}
$$

Notice that $A$ is an $M$ matrix since it has $Z$ pattern, that is, the off-diagonal elements are nonpositive and all the leading principal minors of $A$ are positive with the conditions $\alpha \rho_{v 1}\left(1-\beta_{I 1}\right) r+\zeta<\delta+\varepsilon r+\phi \theta$ and $\mathscr{R}_{0}^{v}<1-(\zeta \phi \theta)$ $\left.(\delta+\varepsilon r+\phi \theta)^{2}\right)$, and moreover, it is clear that $\widehat{G}(X, \mathbf{Y}) \geq 0$. Hence, the above conditions (H1) and (H2) are satisfied, and by Lemma 1 , this completes the proof.

Now, we study the global stability of endemic equilibrium $E_{*}^{v}$ by the geometric approach. A brief outline of this approach can be found in $[30,31]$.

Theorem 4. Suppose $\alpha \rho_{v 1}\left(1-\beta_{I 1}\right) r+\zeta<\delta+\varepsilon r$ and $\zeta<\alpha \rho_{v 2}$ $\left(1-\beta_{I 2}\right) r$. The endemic equilibrium $E_{*}^{v}$ is globally asymptotically stable in $\Omega$ if $\mathscr{R}_{0}^{v}>1$.

Proof. The Jacobian matrix of system (13) is

$$
J=\left[\begin{array}{ccc}
A_{11} & -\zeta \frac{I}{N} & \alpha \rho_{v 2}\left(1-\beta_{I 2}\right) r-\zeta \frac{I}{N} \\
-\phi \theta & A_{22} & -\phi \theta \\
\phi \theta & 0 & A_{33}
\end{array}\right],
$$

with $\quad A_{11}=\alpha \rho_{v 1}\left(1-\beta_{I 1}\right) r-(\delta+\varepsilon r)+\zeta(N-I-V-W /$ $N)-\zeta(I / \quad N)-\phi \theta, \quad A_{22}=-\phi \theta-(\delta+\varepsilon r), \quad$ and $A_{33}=-(\delta+\varepsilon r)$. Then, its associated second additive compound matrix is

$$
J^{[2]}=\left[\begin{array}{ccc}
A_{11}+A_{22} & -\phi \theta & -\alpha \rho_{v 2}\left(1-\beta_{I 2}\right) r+\zeta \frac{I}{N} \\
0 & A_{11}+A_{33} & -\zeta \frac{I}{N} \\
-\phi \theta & -\phi \theta & A_{22}+A_{33}
\end{array}\right] .
$$

We choose the matrix function $P(I, V, W)=$ $\operatorname{diag}(1,(I / W),(I / W))$ and then $P_{f} P^{-1}=\operatorname{diag}(0,(\dot{I} / I)-$ $(\dot{W} / W),(\dot{I} / I)-(\dot{W} / W))$, where matrix $P_{f}$ is obtained by replacing each entry $p_{i j}$ of $P$ by its derivative in the direction of solution of (13). Thus, the matrix $B=P_{f} P^{-1}+P J^{[2]} P^{-1}$ can be written in the block form as follows:

$$
B=\left[\begin{array}{ll}
B_{11} & B_{12} \\
B_{21} & B_{22}
\end{array}\right],
$$

with 


$$
\begin{aligned}
& B_{11}=A_{11}+A_{22}, \\
& B_{12}=\left[-\phi \theta \frac{W}{I},\left(-\alpha \rho_{v 2}\left(1-\beta_{I 2}\right) r+\zeta \frac{I}{N}\right) \frac{W}{I}\right], \\
& B_{21}=\left[0,-\phi \theta \frac{I}{W}\right]^{T}, \\
& B_{22}=\left[\begin{array}{cc}
A_{11}+A_{33}+\frac{\dot{I}}{I}-\frac{\dot{W}}{W} & -\zeta \frac{I}{N} \\
-\phi \theta & A_{22}+A_{33}+\frac{\dot{I}}{I}-\frac{\dot{W}}{W}
\end{array}\right] .
\end{aligned}
$$

Letting $\left(u_{1}, u_{2}, u_{3}\right)$ denote the vector in $\mathbb{R}^{3}$, we define the following norm in $\mathbb{R}^{3}$ :

$$
\left|\left(u_{1}, u_{2}, u_{3}\right)\right|=\max \left\{\left|u_{1}\right|,\left|u_{2}\right|+\left|u_{3}\right|\right\},
$$

and let $\mu$ denote the Lozinskii measure with respect to this norm:

$$
\mu(B)=\lim _{h \longrightarrow 0^{+}} \frac{|I+h B|-1}{h} .
$$

Then, following [38], we have

$$
\mu(B) \leq \max \left\{g_{1}, g_{2}\right\}
$$

in which $g_{1}=\mu_{1}\left(B_{11}\right)+\left|B_{12}\right|$ and $g_{2}=\left|B_{21}\right|+\mu_{1}\left(B_{22}\right)$. Here, $\left|B_{12}\right|$ and $\left|B_{21}\right|$ are matrix norms with respect to $l_{1}$ vector norm and $\mu_{1}$ denotes the Lozinskii measure with respect to $l_{1}$ vector norm. With these definitions, $\mu_{1}\left(B_{11}\right)=$ $A_{11}+A_{22}$ and $\left|B_{12}\right|=(W / I) \max \left\{\phi \theta, \alpha \rho_{v 2}\left(1-\beta_{I 2}\right) r-\right.$ $\zeta(I / N)\}$ since $\alpha \rho_{v 2}\left(1-\beta_{I 2}\right) r>\zeta,\left|B_{21}\right|=\phi \theta(I / W)$, and

$$
\begin{aligned}
\mu_{1}\left(B_{22}\right) & =\max \left\{A_{11}+A_{33}+\frac{\dot{I}}{I}-\frac{\dot{W}}{W}+\phi \theta, A_{22}+A_{33}+\frac{\dot{I}}{I}-\frac{\dot{W}}{W}+\zeta \frac{I}{N}\right\} \\
& =A_{33}+\frac{\dot{I}}{I}-\frac{\dot{W}}{W}+\max \left\{A_{11}+\phi \theta, A_{22}+\zeta \frac{I}{N}\right\} .
\end{aligned}
$$

Then, for $g_{1}$ and $g_{2}$, we have

$$
\begin{aligned}
& g_{1}=\mu_{1}\left(B_{11}\right)+\left|B_{12}\right| \\
& =A_{11}+A_{22}+\frac{W}{I} \max \left\{\alpha \rho_{v 2}\left(1-\beta_{I 2}\right) r-\zeta \frac{I}{N}, \phi \theta\right\} \\
& =\alpha \rho_{v 1}\left(1-\beta_{I 1}\right) r-(\delta+\varepsilon r)+\zeta \frac{N-I-V-W}{N}-\zeta \frac{I}{N}-\phi \theta-\phi \theta-(\varepsilon r+\delta) \\
& +\frac{W}{I} \max \left\{\alpha \rho_{v 2}\left(1-\beta_{I 2}\right) r-\zeta \frac{I}{N}, \phi \theta\right\} \\
& =\frac{\dot{I}}{I}-\alpha \rho_{v 2}\left(1-\beta_{I 2}\right) r \frac{W}{I}-(\varepsilon r+\delta)+\frac{W}{I} \max \left\{\alpha \rho_{v 2}\left(1-\beta_{I 2}\right) r-\zeta \frac{I}{N}-\phi \theta, 0\right\} \\
& =\frac{\dot{I}}{I}-(\varepsilon r+\delta)+\frac{W}{I} \max \left\{-\zeta \frac{I}{N}-\phi \theta,-\alpha \rho_{v 2}\left(1-\beta_{I 2}\right) r\right\} \leq \frac{\dot{I}}{I}-\varepsilon r, \\
& g_{2}=\left|B_{21}\right|+\mu_{1}\left(B_{22}\right) \\
& =\phi \theta \frac{I}{W}+A_{33}+\frac{\dot{I}}{I}-\frac{\dot{W}}{W}+\max \left\{A_{11}+\phi \theta, A_{22}+\zeta \frac{I}{N}\right\} \\
& =\phi \theta \frac{I}{W}-(\delta+\varepsilon r)+\frac{\dot{I}}{I}-\frac{\dot{W}}{W}+\max \left\{\alpha \rho_{v 1}\left(1-\beta_{I 1}\right) r-(\delta+\varepsilon r)\right. \\
& \left.+\zeta \frac{N-I-V-W}{N}-\zeta \frac{I}{N},-\phi \theta-(\delta+\varepsilon r)+\zeta \frac{I}{N}\right\} \\
& =\phi \theta \frac{I}{W}+\frac{\dot{I}}{I}-\left(\phi \theta \frac{I}{W}-(\varepsilon r+\delta)\right)-(\varepsilon r+\delta) \\
& +\max \left\{\alpha \rho_{v 1}\left(1-\beta_{I 1}\right) r-(\delta+\varepsilon r)+\zeta \frac{N-I-V-W}{N}-\zeta \frac{I}{N},-\phi \theta-(\delta+\varepsilon r)+\zeta \frac{I}{N}\right\} \\
& =\frac{\dot{I}}{I}+\max \left\{\alpha \rho_{v 1}\left(1-\beta_{I 1}\right) r-(\delta+\varepsilon r)+\zeta \frac{N-I-V-W}{N}-\zeta \frac{I}{N},-\phi \theta-(\delta+\varepsilon r)+\zeta \frac{I}{N}\right\} \\
& \leq \frac{\dot{I}}{I}-\min \left\{(\delta+\varepsilon r)-\alpha \rho_{\nu 1}\left(1-\beta_{I 1}\right) r-\zeta, \phi \theta+(\delta+\varepsilon r)-\zeta\right\} \\
& \leq \frac{\dot{I}}{I}-b
\end{aligned}
$$


where $\quad b=\min \left\{(\delta+\varepsilon r)-\alpha \rho_{v 1}\left(1-\beta_{I 1}\right) r-\zeta, \phi \theta+(\delta+\varepsilon r)\right.$ $-\zeta\}$ is a positive constant since $\alpha \rho_{v 1}\left(1-\beta_{I 1}\right) r+\zeta<\delta+\varepsilon r$ and $\zeta<\phi \theta+\delta+\varepsilon r$. Consequently, $\mu(B) \leq(I / I)-\bar{b}$, where $\bar{b}=\min \{b, \varepsilon r\}>0$. Along each solution $(I(t), V(t), W(t))$ of (10) with $(I(t), V(t), W(t)) \in \Gamma$, we thus have

$$
\frac{1}{t} \int_{0}^{t} \mu(B) \mathrm{d} s \leq \frac{1}{t} \int_{0}^{t}\left(\frac{\dot{I}}{I}-\bar{b}\right) \mathrm{d} s=\frac{1}{t} \ln \frac{I(t)}{I(0)}-\bar{b},
$$

which implies that

$$
\bar{q}_{2}:=\limsup _{t \longrightarrow \infty} \frac{1}{t} \int_{0}^{t} \mu(B) \mathrm{d} s \leq-\frac{\bar{b}}{2}<0,
$$

for any $(S(0), I(0), V(0), W(0)) \in \Omega$. By the results in $[30,31]$, the unique equilibrium $E_{*}^{v}$ is globally asymptotically stable in $\Omega$ if $\bar{q}_{2}<0$. This completes the proof.

Remark 1. If $\mathscr{R}_{0}^{v}>1+\left(\zeta(\delta+\varepsilon r) /(\delta+\varepsilon r+\phi \theta)^{2}\right)$, susceptible population will decay towards a negative point where the entire population will become infected. In this situation, we assume that all the populations are infected. It is the worst case that farm managements do not expect it happen.

\section{Results and Discussion}

We present the numerical simulation of the proposed model to verify the analytical results. A fourth-order Runge-Kutta numerical method is used. Parameter values are provided in Table 1 either by consulting previous literatures $[18,25,26,28]$ or by estimating from distributions. The external infection source $\sigma$ is set to zero in all cases except the discussion in Section 4.3 where the impact of external infection is investigated. Assume the parameter $\eta$ obeys uniform distribution $U(0.3,0.9)$, so the mean value is 0.6 as indicated in Table 1. Similarly, parameters $c$, $\phi$, and $\rho_{v 2}$ obey uniform distribution $U(0.4,1), \quad U(0.25,0.75)$, and $U(0.2,0.6)$, respectively. In particular, steady-state information of six-equation system (1) is incorporated in estimating $p, q$, and $r$. That is the proportions of offspring, heifers, and cows are estimated from the steady state in (1) which results in $S_{O}: S_{H}: S_{C}=1: 1: 2$ and $I_{O}: I_{H}: I_{C}=1: 1: 2$. To verify the reliability of model reduction, dynamics of all states obtained by the six-equation model (1) are presented in Figure 2. A case with $70 \%$ initial prevalence demonstrates that reduced model (3) possesses the same prevalence $27 \%$ at the endemic state and thus shows the feasibility of model reduction.

Dynamics of the baseline case shown in Figure 3 verify Theorem 1. When $\mathscr{R}_{0}=0.7868, E_{0}=(N, 0)$ is the unique equilibrium and $N$. caninum dies out from farm. That different initial prevalence decays to $E_{0}$ and evidences the global stability numerically. When $\mathscr{R}_{0}=1.1624$, $E_{*}=\left(S^{*}, I^{*}\right)$ is the only endemic equilibrium and $N$. caninum persists with $27 \%$ prevalence. The baseline case, which does not include any additional control measure targeting N. caninum transmission, was considered as a reference.

4.1. Global Uncertainty and Sensitivity Analysis. To evaluate the effect of parameters on the basic reproduction number $\mathscr{R}_{0}$ and control production numbers $\mathscr{R}_{0}^{m}, \mathscr{R}_{0}^{t}$, and $\mathscr{R}_{0}^{v}$, we performed global uncertainty and sensitivity analysis. Each parameter is assigned a probability distribution or being varied uniformly within $20 \%$ of its value in Table 1 and the parameter sample size is 1000 .

In the baseline case shown in Figure 4(a), most realizations of $\mathscr{R}_{0}(96 \%)$ maintain being larger than unity towards varied environments. This confirms the biological observation that $N$. caninum is endemic and could rarely die out in cattle populations without intervention. The frequency distribution of $\mathscr{R}_{0}$ ranges from 0.95 to 1.35 with a mean value of 1.1154 which indicates that an infected animal may infect 1 or 2 susceptible animals during the infectious period. Figures 4(b)-4(d) depict frequency distributions of $\mathscr{R}_{0}^{m}$ (medication), $\mathscr{R}_{0}^{t}$ (test-and-cull), and $\mathscr{R}_{0}^{v}$ (vaccination), respectively. With the chemotherapy (see Figure 4(b)), frequency distribution of $\mathscr{R}_{0}^{m}$ shifts left less than 1 with mean value 0.5916 . Strategy by test-based culling (see Figure 4(c)) is however more effective with $\mathscr{R}_{0}^{t}$ ranging from 0.2 to 0.5 and the average is 0.3284 . Control of taking vaccine continuously (see Figure 4(d)) is the most stable option with the least varied range of control reproduction number. Moreover, vaccination is also effective with mean value 0.6579 ranging from 0.28 to 0.72 . The analysis reveals that three controls are all effective considering controlling the $N$. caninum spreading among dairy. Furthermore, control strategies of test-and-cull and vaccination are much more stable concerning the uncertain environment which confirms the conclusion given in [26].

Sensitivity analysis investigates the impact of parameter uncertainties on the overall output. Pearson correlation coefficient (PEAR) is used to quantify the effect of parameters on reproduction numbers in baseline and control cases in Figure 5. Without any intervention, the pregnancy rate $\alpha$, vertical infection rate $\rho_{v 1}$, and mortality rate $\delta$ play an important role in determining the basic reproduction number $\mathscr{R}_{0}$. With treatment, the recovery rate $\eta$ is the most crucial parameter and $\rho_{v 1}$ becomes less effective since treatment on offspring born from the infected dam partially blocked the vertical infection route. When test-and-cull or vaccination is applied, the vertical transmission rate $\rho_{v 1}$ also shows less impact. This is explained that with test-based culling, seropositive individuals have been culled, and thus, the risk of vertical infection is reduced. With the continuous vaccination, a portion of successfully vaccinated animals will be protected from N. caninum, and this portion has a lower risk of passing the disease to their daughters. Thus, $\rho_{v 1}$ is observed to be less effective and the vertical infection rate of vaccinated infected cow $\rho_{v 2}$ becomes the most influential parameter. It is noticed that the control reproduction number highly depends on the parameters related to control 


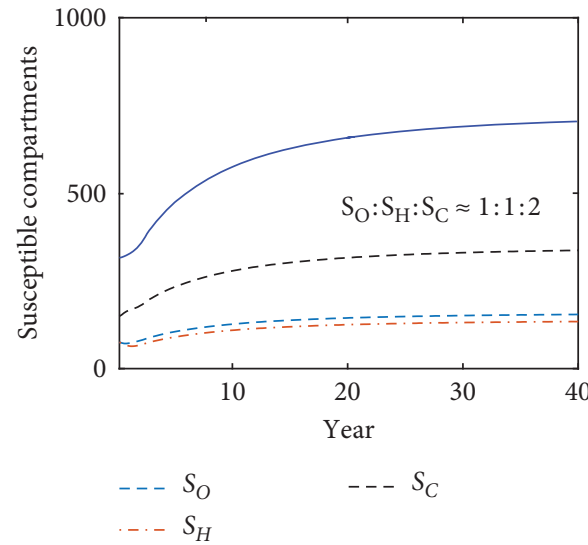

(a)

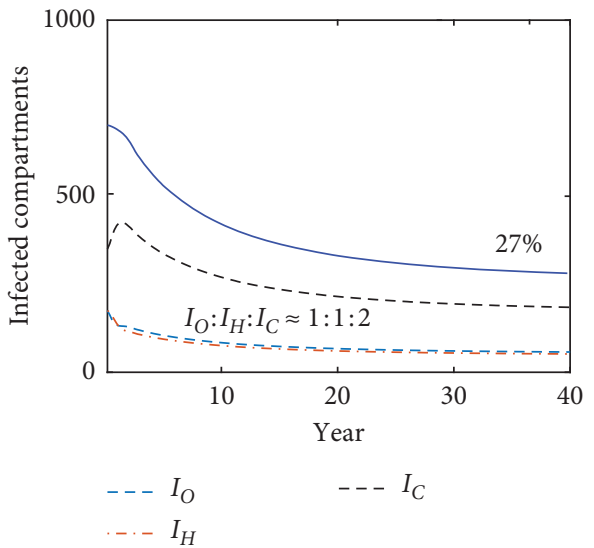

(b)

Figure 2: Dynamics of the six-equation model (1) with $70 \%$ initial prevalence. The final steady state (in solid blue line) prevalence is about $27 \%$ where the proportion of three states, offspring $(O)$, heifers $(H)$, and cows $(C)$, is about 1: 1: 2 in both susceptible and infected categories.

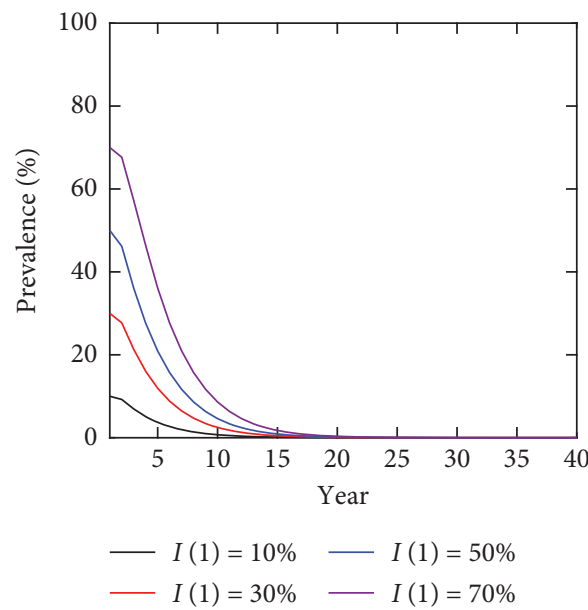

(a)

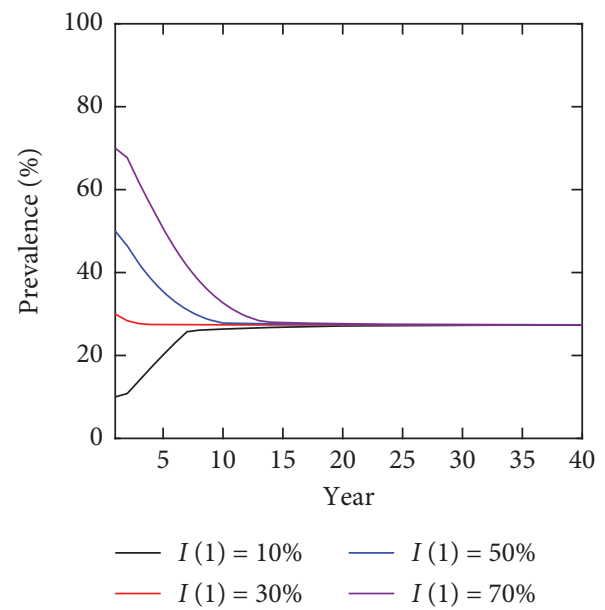

(b)

FIGURE 3: Global stability of disease-free and endemic equilibria. (a) Reproduction number $\mathscr{R}_{0}=0.7868$. The vertical infection rate $\rho_{v 1}$ is set to 0.5 and all other parameters not specified are used according to Table 1. (b) Reproduction number $\mathscr{R}_{0}=1.1624$.

actions in the three measures. In particular, the recovery rate of dams $\eta$ and culling proportion $c$ are the most influential parameters in medication and test-and-cull, respectively. Whereas, in vaccination strategy, vaccination coverage rate $\phi$ is most significant except the vertical transmission rate of infected $\operatorname{cow} \rho_{v 1}$ and the vertical infection rate of vaccinated infected cow $\rho_{v 2}$.

4.2. Effectiveness of Control Strategies. Evaluation of control options can focus on the effect on control reproduction number which helps to gain an insight into the effectiveness. Using expressions (6), (7), and (12), we discuss the effectiveness of medication, test-and-cull, and vaccination based on the performance of $\mathscr{R}_{0}^{m}, \mathscr{R}_{0}^{t}$, and $\mathscr{R}_{0}^{v}$. In Figure 6, the reproduction numbers of different controls are plotted against different parameters induced by additional management action. The parameters include the recovery rate of dams $\eta$, the culling rate of seropositive individuals $c$, the vertical infection rate of vaccinated infected cow $\rho_{v 2}$, and the coverage of vaccination $\phi$ while these factors have shown a major impact in the sensitivity analysis (see Figure 5). When medication is applied, the influential parameter, the recovery rate of offsprings $\eta$, appears as a reduction term multiplied to the vertical transmission route. Figure 6(a) illustrates that $\mathscr{R}_{0}^{m}$ decreases over $\eta$, while the lowest efficacy to prevent vertical transmission is around 0.4 to maintain the effectiveness of treatment. Additional management actions, testing and culling, are taken in the test-and-cull control strategy. Similarly, Figure $6(\mathrm{~b})$ shows $\mathscr{R}_{0}^{t}$ decreases over the culling proportion $c$, and the lowest culling rate around 0.3 is suggested. With the implementation of taking vaccine, the reproduction number $\mathscr{R}_{0}^{v}$ almost maintains lower than 1 except with a rather low vaccination coverage rate $\phi$ (around 0.1). 


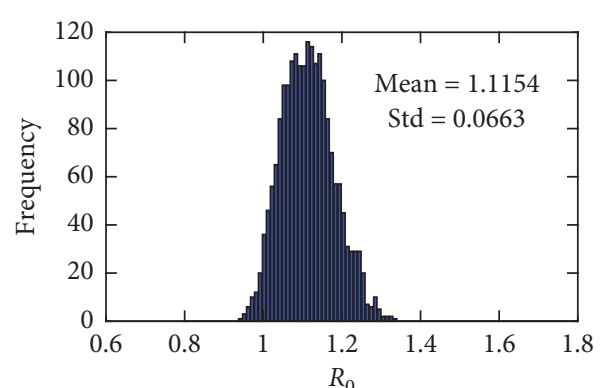

(a)

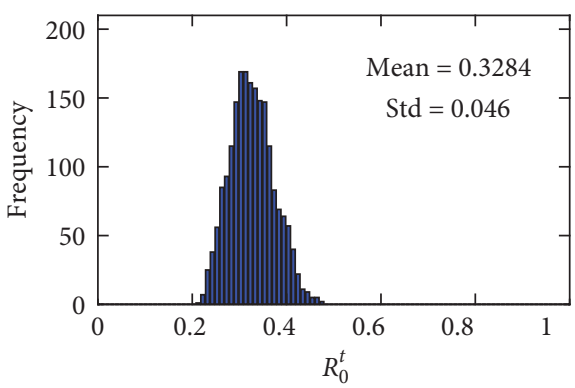

(c)

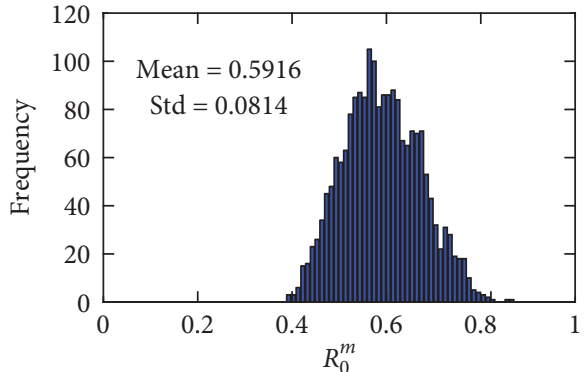

(b)

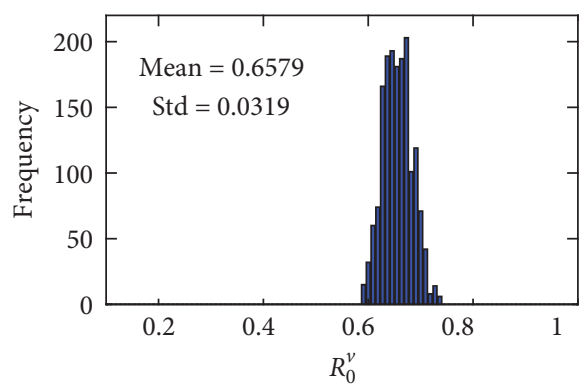

(d)

FIGURE 4: Uncertainty analysis of reproduction number $\mathscr{R}_{0}, \mathscr{R}_{0}^{m}, \mathscr{R}_{0}^{t}$, and $\mathscr{R}_{0}^{v}$. Frequency distribution of reproduction number over 1000 simulations with varied parameters. (a) Baseline case. (b) Medication. (c) Test-and-cull. (d) Vaccination.

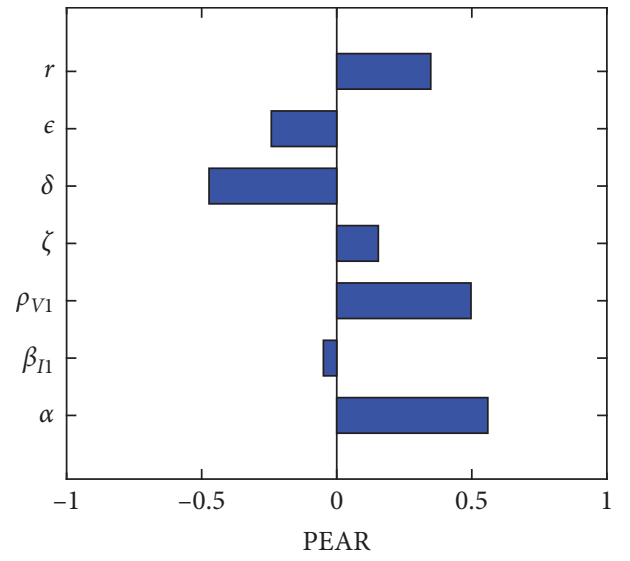

(a)

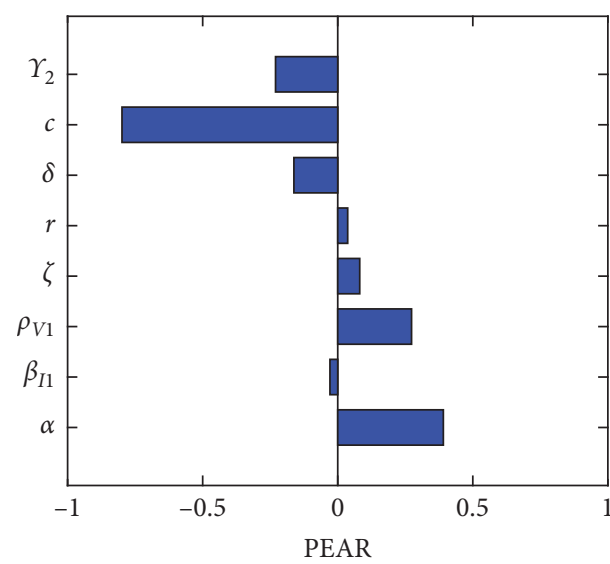

(c)

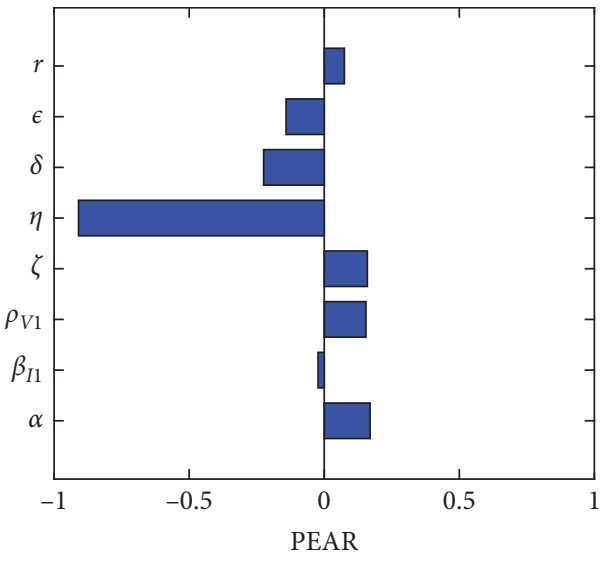

(b)

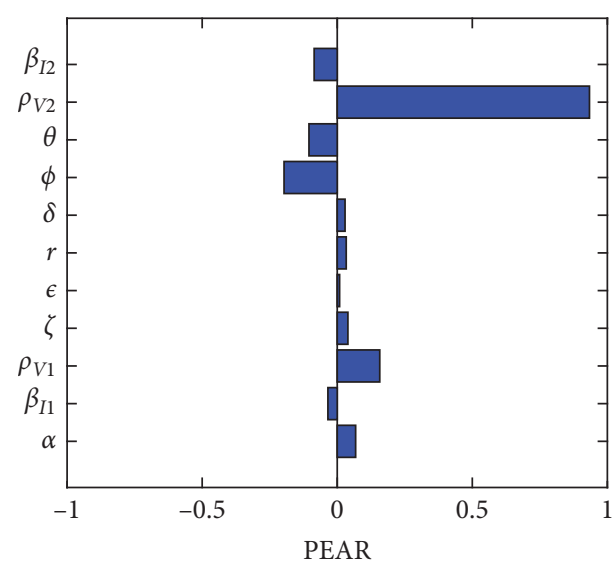

(d)

Figure 5: Parameter sensitivity analysis using Person correlation coefficient (PEAR) in (a) baseline case and three control scenarios, (b) medication, (c) test-and-cull, And (d) vaccination. 


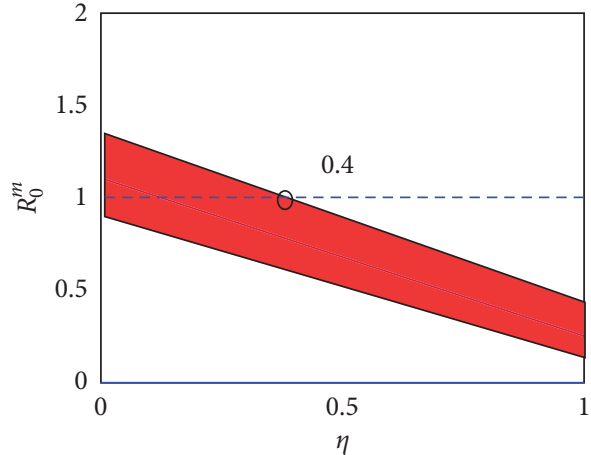

(a)

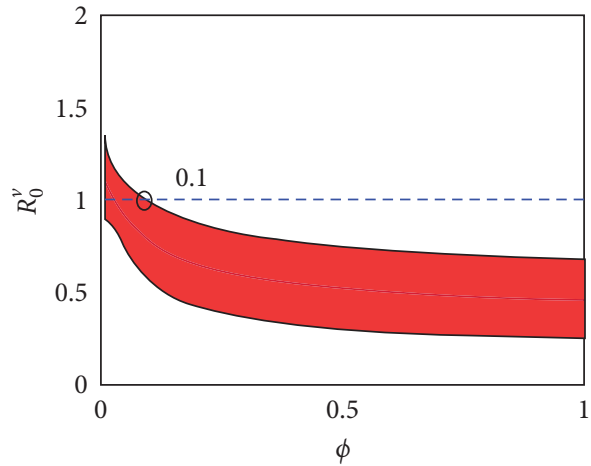

(c)

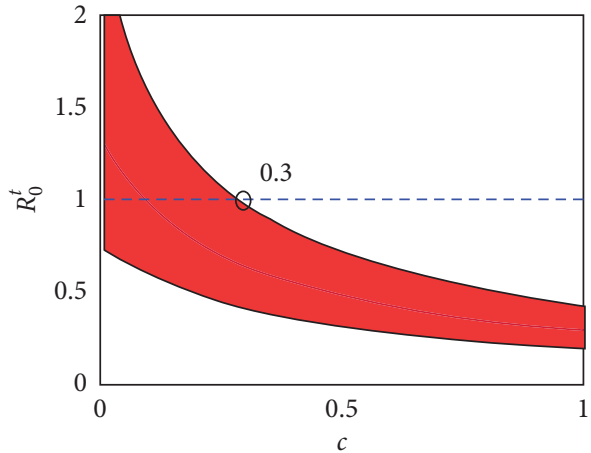

(b)

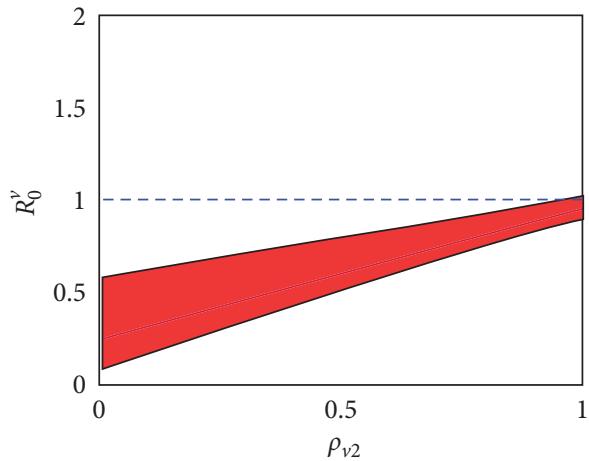

(d)

Figure 6: Dynamics of control reproduction number versus key factors. All parameters are varied uniformly within $20 \%$ of its value in Table 1 except $\sigma=0$. Red region denotes the area where the 1000 simulation realizations lie. (a) $\mathscr{R}_{0}^{m}$ versus the recovery rate $\eta$. (b) $\mathscr{R}_{0}^{t}$ versus culling proportion c. (c) $\mathscr{R}_{0}^{v}$ versus coverage of vaccination $\phi$. (d) $\mathscr{R}_{0}^{v}$ versus the vertical infection rate of vaccinated infected cows $\rho_{v 2}$.

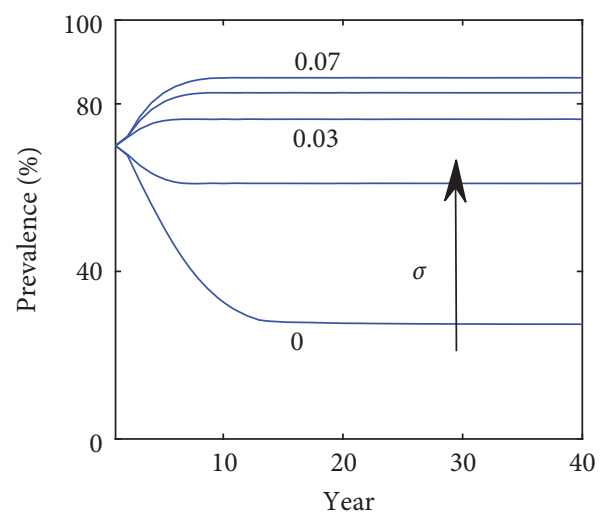

(a)

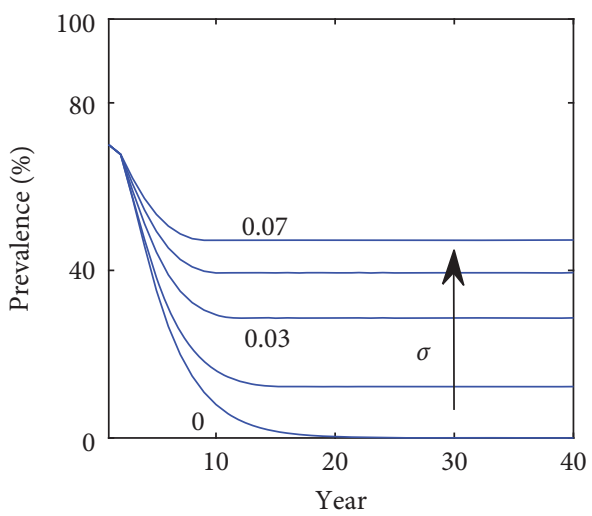

(b)

FIGURE 7: Continued. 


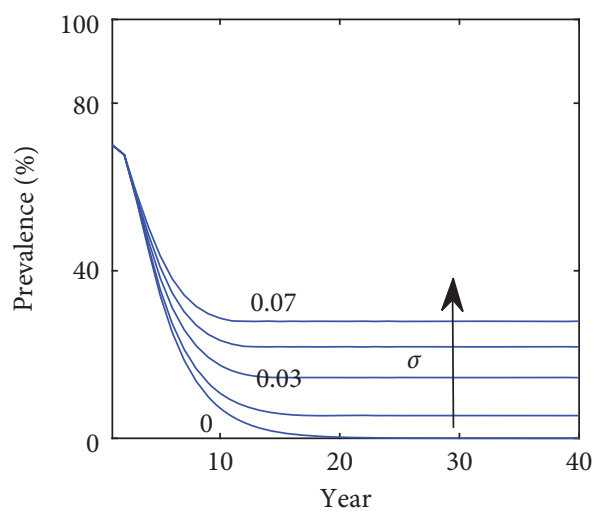

(c)

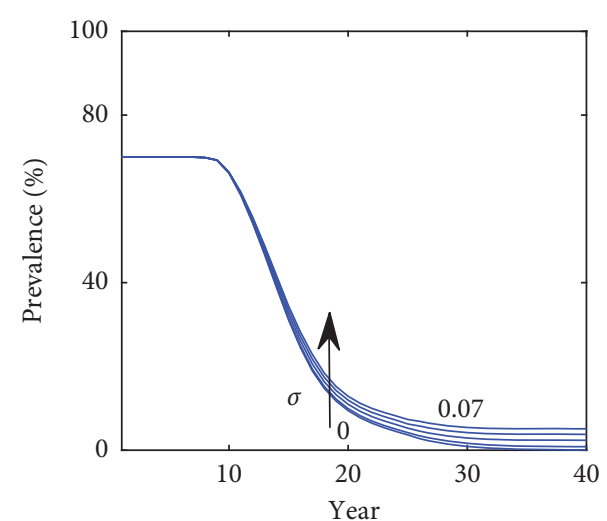

(d)

Figure 7: Dynamics of prevalence without $(\sigma=0)$ or with external infection source $(\sigma=0.01,0.03,0.05,0.07)$ in baseline case: (a) three control cases, (b) medication, (c) test-and-cull, and (d) vaccination. Arrow shows $\sigma$ increases from 0, 0.01, 0.03, and 0.05 to 0.07 , and other parameters are used according to Table 1.

Although the vertical transmission rate of vaccinated infected cows $\rho_{v 2}$ shows a major impact on $\mathscr{R}_{0}^{v}$ according to uncertainty analysis, the effectiveness of $\mathscr{R}_{0}^{v}$ rarely depends on $\rho_{v 2}$ as illustrated in Figure 6(d). Figures 6(c) and 6(d) evidence that vaccination is the most stable and effective option due to the superiority in reducing the risk of abortion and preventing horizontal transmission simultaneously.

4.3. Impact of External Source of Infection. External infections of $N$. caninum may arise from other hosts outside the herd. For example, dogs can shed oocysts of N. caninum and contaminate the environment. In reality, external sources of transmission may be hard to eliminate especially for a large farm or farm with poor biosecurity. At present, no reliable value can be given to external transmission parameter $\sigma$ based on either field or experimental data, and thus, $\sigma \in[0,0.07]$ is at least plausible referred to [16]. Figure 7 illustrates the effect of varied external source of contamination $(\sigma=0,0.01,0.03,0.05,0.07)$ on reducing prevalence. At $\sigma=0$, the disease diminishes to zero with the help of three measures. When the outside-herd level transmission increases from 0 to 0.07 , within-herd prevalence moves away from the disease-free state in test-and-cull and medication cases (the same in the baseline case). Whereas, with vaccine dose, the disease-free state shifts slightly upward. The external source of infection has relatively less impact on the persistence in the vaccination control strategy.

The presence of external sources of infection can increase the prevalence and reduce control efficiency. Even in the disease-free state, small external sources of infection can cause a significant growth of prevalence, especially in medication and test-and-cull scenarios. This reminds us that test-based culling of seropositive animals and medical treatment on offspring should be done only if the external sources of transmission by Neospora-oocysts are prevented. Overall, for farms with good biosecurity where other hosts outside herd are prevented, medication, test-and-cull, and vaccination will be effective measures. However, once this condition is not satisfied, these potential controls lose the ability to eliminate the disease.

\section{Conclusion}

In this research, we develop the epidemiological model for $N$. caninum transmission in cattle without control or with a single control strategy of medication, test-and-cull, or vaccination. The system of six-equation is reduced to a twoequation model where the most influential indicator, the basic reproduction number, is derived. Using the analytical solution or geometric approach, we have investigated the global asymptotic stability of the disease-free and endemic equilibria. Uncertainty analysis demonstrates that $N$. caninum could rarely die out without intervention. All three control strategies, including medication, test-and-cull, and vaccination, are verified to be effective even with the varied dairy management or environment, and more importantly, vaccination is the most stable measure towards the changeable inputs. This simultaneously confirms the results in the previous study [26]. Sensitivity analysis with respect to input parameters reveals the key factors which mainly lie in parameters related to additional management-related actions. Furthermore, dynamics of control reproduction number against these key factors suggest the lowest efficacy to achieve effectiveness, such as adopting medication with medicine efficacy higher than 0.4 to prevent vertical transmission or implementing test-and-cull with culling coverage larger than 0.3. Vaccination, however, provides a rather loose constraint that the vaccine covering rate higher than 0.1 is enough to keep control effective. Our analysis of external infection has shown that rather a low level of external infection is important since the disease could change from extinction to persistence. It suggests that $N$. caninum transmission can be effectively controlled by medicine treatment, selected culling of seropositive cows, or vaccination, provided external sources of infection are controlled. Preventive measures should at least include the prevention of access of other hosts, such as dogs, to cattle by strict hygiene measures. Otherwise, these controls lose effectiveness.

The dynamical model established in this study focuses on a single farm which is assumed to be homogeneous mixing. Whereas, the spatial motions of animals are also important 
for controlling the disease. Therefore, it is necessary to consider the diffusion of animals during the disease spread which will lead to the partial differential equations $[39,40,41,42,43]$. This issue will be well explored in the future study.

\section{Data Availability}

The parameter values used to support the findings of this study are included within the article.

\section{Conflicts of Interest}

The authors declare no conflicts of interest.

\section{Acknowledgments}

This work was supported by CityU Strategic Research Grants (Project nos. CityU 11303719 and CityU 11301520). Yue Liu would like to thank the University Grants Committee (UGC) in Hong Kong for the financial support.

\section{References}

[1] W. Wouda, J. P. Dubey, and M. C. Jenkins, "Serological diagnosis of bovine fetal neosporosis," The Journal of Parasitology, vol. 83, no. 3, pp. 545-547, 1997.

[2] J. P. Dubey, "Review of Neospora caninum and neosporosis in animals," The Korean Journal of Parasitology, vol. 41, no. 1, pp. 1-16, 2003.

[3] D. J. Wilson, K. Orsel, J. Waddington et al., "Neospora caninum is the leading cause of bovine fetal loss in British Columbia, Canada," Veterinary Parasitology, vol. 218, pp. 46-51, 2016.

[4] J. Paré, M. C. Thurmond, and S. K. Hietala, "Congenital Neospora caninum infection in dairy cattle and associated calfhood mortality," Canadian Journal of Veterinary Research, vol. 60, no. 2, pp. 133-139, 1996.

[5] H. C. Davison, A. Otter, and A. J. Trees, "Estimation of vertical and horizontal transmission parameters of Neospora caninum infections in dairy cattle," International Journal for Parasitology, vol. 29, no. 10, pp. 1683-1689, 1999.

[6] C. J. M. Bartels, I. Huinink, M. L. Beiboer et al., "Quantification of vertical and horizontal transmission of Neospora caninum infection in Dutch dairy herds," Veterinary Parasitology, vol. 148, no. 2, pp. 83-92, 2007.

[7] H.-P. Fuehrer, I. Blöschl, C. Siehs, and A. Hassl, "Detection of Toxoplasma gondii, Neospora caninum, and Encephalitozoon cuniculi in the brains of common voles (Microtus arvalis) and water voles (Arvicola terrestris) by gene amplification techniques in western Austria (Vorarlberg)," Parasitology Research, vol. 107, no. 2, pp. 469-473, 2010.

[8] R. Atkinson, R. Cook, L. Reddacliff et al., "Seroprevalence of Neospora caninum infection following an abortion outbreak in a dairy cattle herd," Australian Veterinary Journal, vol. 78, no. 4, pp. 262-266, 2000.

[9] D. U. Pfeiffer, N. B. Williamson, M. P. Reichel, J. J. Wichtel, and W. R. Teague, "A longitudinal study of Neospora caninum infection on a dairy farm in New Zealand," Preventive Veterinary Medicine, vol. 54, no. 1, pp. 11-24, 2002.

[10] J. Hernandez, C. Risco, and A. Donovan, "Association between exposure to Neospora caninum and milk production in dairy cows," Journal of the American Veterinary Medical Association, vol. 219, no. 5, pp. 632-635, 2001.

[11] J. P. Dubey and D. S. Lindsay, "A review of Neospora caninum and neosporosis," Veterinary Parasitology, vol. 67, no. 1-2, pp. 1-59, 1996.

[12] M. P. Reichel, M. Alejandra Ayanegui-Alcérreca, L. F. P. Gondim, and J. T. Ellis, "What is the global economic impact of Neospora caninum in cattle-the billion dollar question," International Journal for Parasitology, vol. 43, no. 2, pp. 133-142, 2013.

[13] C. Li, Z.-G. Guo, and Z.-Y. Zhang, “Transmission dynamics of a brucellosis model: basic reproduction number and global analysis," Chaos, Solitons \& Fractals, vol. 104, pp. 161-172, 2017.

[14] A. Abidemi, M. I. Abd Aziz, and R. Ahmad, "Vaccination and vector control effect on dengue virus transmission dynamics: modelling and simulation," Chaos, Solitons \& Fractals, vol. 133, Article ID 109648, 2020.

[15] J. Liang, Z. Zhao, and C. Li, "Rich dynamics of a brucellosis model with transport," Complexity, vol. 2020, Article ID 5050393, 7 pages, 2020.

[16] N. P. French, D. Clancy, H. C. Davison, and A. J. Trees, "Mathematical models of Neospora caninum infection in dairy cattle: transmission and options for control," International Journal for Parasitology, vol. 29, no. 10, pp. 1691-1704, 1999.

[17] R. L. Larson, D. K. Hardin, and V. L. Pierce, "Economic considerations for diagnostic and control options forNeospora caninum-induced abortions in endemically infected herds of beef cattle," Journal of the American Veterinary Medical Association, vol. 224, no. 10, pp. 1597-1604, 2004.

[18] B. Häsler, K. D. C. Stärk, H. Sager, B. Gottstein, and M. Reist, "Simulating the impact of four control strategies on the population dynamics of Neospora caninum infection in Swiss dairy cattle," Preventive Veterinary Medicine, vol. 77, no. 3-4, pp. 254-283, 2006.

[19] M. P. Reichel and J. T. Ellis, "If control of Neospora caninum infection is technically feasible does it make economic sense?" Veterinary Parasitology, vol. 142, no. 1-2, pp. 23-34, 2006.

[20] C. A. Hall, M. P. Reichel, and J. T. Ellis, "Neospora abortions in dairy cattle: diagnosis, mode of transmission and control," Veterinary Parasitology, vol. 128, no. 3-4, pp. 231-241, 2005.

[21] L. V. de Aquino Diniz, A. F. Minutti, B. de Souza Lima Nino et al., "Vertical transmission of Neospora caninum in bovine fetuses from a slaughterhouse in Brazil," Tropical Animal Health and Production, vol. 51, no. 6, pp. 1751-1755, 2019.

[22] A. R. Moen, W. Wouda, M. F. Mul, E. A. M. Graat, and T. van Werven, "Increased risk of abortion following Neospora caninum abortion outbreaks: a retrospective and prospective cohort study in four dairy herds," Theriogenology, vol. 49, no. 7, pp. 1301-1309, 1998.

[23] H. C. Davison, C. S. Guy, J. W. McGarry et al., "Experimental studies on the transmission of Neospora caninum between cattle," Research in Veterinary Science, vol. 70, no. 2, pp. 163-168, 2001.

[24] T. Dijkstra, H. W. Barkema, J. W. Hesselink, and W. Wouda, "Point source exposure of cattle to Neospora caninum consistent with periods of common housing and feeding and related to the introduction of a dog," Veterinary Parasitology, vol. 105, no. 2, pp. 89-98, 2002.

[25] S. Schärrer, P. Presi, J. Hattendorf, N. Chitnis, M. Reist, and J. Zinsstag, "Demographic model of the Swiss cattle population for the years 2009-2011 stratified by gender, age and production type," PLoS One, vol. 9, no. 10, Article ID e109329, 2014. 
[26] Y. Liu, M. P. Reichel, and W.-C. Lo, "Combined control evaluation for Neospora caninum infection in dairy: economic point of view coupled with population dynamics," Veterinary Parasitology, vol. 277, Article ID 108967, 2020.

[27] P. van den Driessche and J. Watmough, "Reproduction numbers and sub-threshold endemic equilibria for compartmental models of disease transmission," Mathematical Biosciences, vol. 180, no. 1-2, pp. 29-48, 2002.

[28] B. Häsler, G. Regula, K. D. C. Stärk, H. Sager, B. Gottstein, and M. Reist, "Financial analysis of various strategies for the control of Neospora caninum in dairy cattle in Switzerland," Preventive Veterinary Medicine, vol. 77, no. 3-4, pp. 230-253, 2006.

[29] Y. Liu and W.-C. Lo, "Stability analysis and optimal control of production-limiting disease in farm with two vaccines," Discrete \& Continuous Dynamical Systems-B, 2021.

[30] M. Y. Li and J. S. Muldowney, "A geometric approach to global-stability problems," SIAM Journal on Mathematical Analysis, vol. 27, no. 4, pp. 1070-1083, 1996.

[31] M. Y. Li, H. L. Smith, and L. Wang, "Global dynamics of an SEIR epidemic model with vertical transmission," SIAM Journal on Applied Mathematics, vol. 62, no. 1, pp. 58-69, 2001.

[32] S. Kritzner, H. Sager, J. Blum, R. Krebber, G. Greif, and B. Gottstein, "An explorative study to assess the efficacy of Toltrazuril-sulfone (Ponazuril) in calves experimentally infected with Neospora caninum," Annals of Clinical Microbiology and Antimicrobials, vol. 1, no. 1, p. 4, 2002.

[33] B. Gottstein, G. R. Razmi, P. Ammann, H. Sager, and N. Müller, "Toltrazuril treatment to control diaplacentalNeospora caninumtransmission in experimentally infected pregnant mice," Parasitology, vol. 130, no. 1, pp. 41-48, 2005.

[34] C. J. M. Bartels, G. van Schaik, J. P. Veldhuisen, B. H. P. van den Borne, W. Wouda, and T. Dijkstra, "Effect of Neospora caninum-serostatus on culling, reproductive performance and milk production in Dutch dairy herds with and without a history of Neospora caninum-associated abortion epidemics," Preventive Veterinary Medicine, vol. 77, no. 3-4, pp. 186-198, 2006.

[35] C. Castillo-Chavez, Z. Feng, and W. Huang, "On the computation of R 0 and its role on global stability," in Mathematical Approaches for Emerging and Reemerging Infectious Disease: An Introduction Springer-Verlag, New York, NY, USA, 2002.

[36] J. P. Tian and J. Wang, "Global stability for cholera epidemic models," Mathematical Biosciences, vol. 232, no. 1, pp. 31-41, 2011.

[37] K. Chakraborty, S. Jana, and T. K. Kar, "Global dynamics and bifurcation in a stage structured prey-predator fishery model with harvesting," Applied Mathematics and Computation, vol. 218, no. 18, pp. 9271-9290, 2012.

[38] R. H. Martin, "Logarithmic norms and projections applied to linear differential systems," Journal of Mathematical Analysis and Applications, vol. 45, no. 2, pp. 432-454, 1974

[39] L. Zhang, Z.-C. Wang, and X.-Q. Zhao, "Threshold dynamics of a time periodic reaction-diffusion epidemic model with latent period," Journal of Differential Equations, vol. 258, no. 9, pp. 3011-3036, 2015.

[40] G. Viglialoro, "Global existence in a two-dimensional chemotaxis-consumption model with weakly singular sensitivity," Applied Mathematics Letters, vol. 91, pp. 121-127, 2019.

[41] T. Li, N. Pintus, and G. Viglialoro, "Properties of solutions to porous medium problems with different sources and boundary conditions," Zeitschrift für angewandte Mathematik und Physik, vol. 70, no. 3, pp. 1-18, 2019.

[42] T. Li and G. Viglialoro, "Boundedness for a nonlocal reaction chemotaxis model even in the attraction-dominated regime," 2020, http://arxiv.org/abs/2004.10991.

[43] G. Viglialoro and T. E. Woolley, "Solvability of a Keller-Segel system with signal-dependent sensitivity and essentially sublinear production," Applicable Analysis, vol. 99, no. 14, pp. 2507-2525, 2019. 